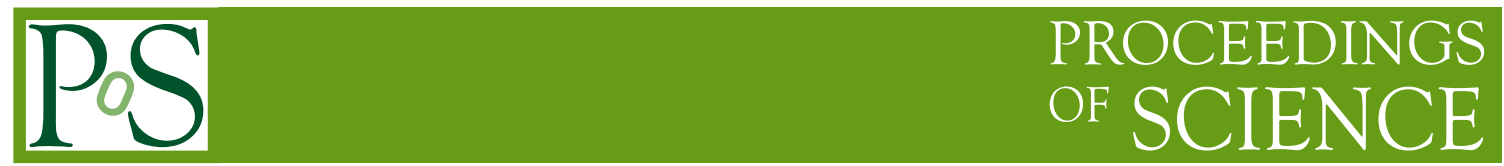

\title{
Highlights from the ALICE collaboration
}

\author{
David Dobrigkeit Chinellato for the ALICE Collaboration ${ }^{a, *}$ \\ ${ }^{a}$ Instituto de Física Gleb Wataghin, \\ R. Sérgio Buarque de Holanda, 777, Campinas, São Paulo, Brazil \\ E-mail: daviddc@ifi.unicamp.br
}

\begin{abstract}
Significant advances in heavy-ion physics have come about as almost ten years have passed since the beginning of the heavy-ion programme at the LHC. In this document, we review some key results from the ALICE collaboration on a variety of topics, from properties of the quark-gluon plasma (QGP) to basic QCD, and discuss how these set the stage for further measurements in the next decade.
\end{abstract}

40th International Conference on High Energy physics - ICHEP2020

July 28 - August 6, 2020

Prague, Czech Republic (virtual meeting)

\footnotetext{
${ }^{*}$ Speaker
} 


\section{Introduction}

The primary mission of the ALICE collaboration is the study of the properties of the quarkgluon plasma (QGP) created in relativistic heavy-ion collisions [1]. In doing so, the collaboration accesses matter whose behaviour is dictated by QCD at extremely high densities, similar to those thought to have been at play during the very early stages of the universe. Several phenomena appear in such conditions and have been systematically probed by the collaboration, leading to remarkable advances in our understanding of the QGP. These advances can be broadly classified into three broad categories: (a) the study of the basic properties of the QGP, (b) the determination of under which conditions high-density signatures such as collective behaviour and strangeness enhancement develop, which includes also measurements performed in proton-proton $(\mathrm{pp})$ and proton-lead $(\mathrm{p}-\mathrm{Pb})$ collisions and (c) employing the outstanding particle identification capabilities of ALICE to explore many other QCD topics and phenomena. These three classes of studies will be discussed in the next sections.

\section{Properties of the quark-gluon plasma}

A fundamental property of the quark-gluon plasma is that it conveys a strong collective motion broadly denoted as 'flow' to the emitted hadrons. This phenomenon has been studied in great detail using Fourier decompositions of the azimuthal particle distribution. The leading non-trivial component, the elliptic flow $v_{2}$, has been observed in $\mathrm{Pb}-\mathrm{Pb}$ collisions at $\sqrt{s_{N N}}=5.02 \mathrm{TeV}$ for a variety of light-flavour particles, ranging from $\pi$ to heavier objects such as deuterons or ${ }^{3} \mathrm{He}$ [2-4], as can be seen in Fig. 1. In all cases, $v_{2}$ has been shown to exhibit mass ordering at low momenta, which is expected from hydrodynamic models [5]. Moreover, the $v_{2}$ of hadrons containing heavyflavour quarks has also been measured and has been observed to be non-zero for D mesons, $\mathrm{J} / \psi$ and even beauty decay electrons $[6,7]$. Heavier states such as the $\Upsilon(1 \mathrm{~S})$, however, exhibit $v_{2}$ consistent with zero within uncertainties in the measured momentum range [8], which indicates that either they do not participate in the collective motion or they do so only outside of the measured range due to their very large masses.

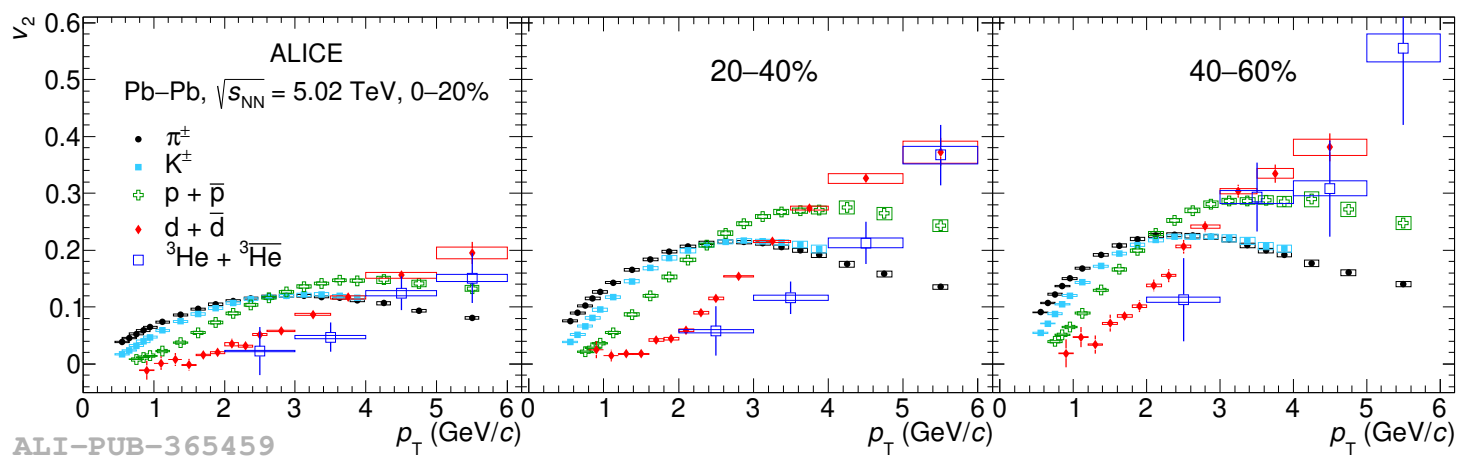

Figure 1: Elliptic flow as a function of $p_{\mathrm{T}}$ for $\pi, \mathrm{K}, \mathrm{p}, \mathrm{d}$ and ${ }^{3} \mathrm{He}[2,3]$.

The motion of the system not only confers linear momentum but also angular momentum to the emitted hadrons. This is a consequence of the fact that in a semi-central collision, the system 


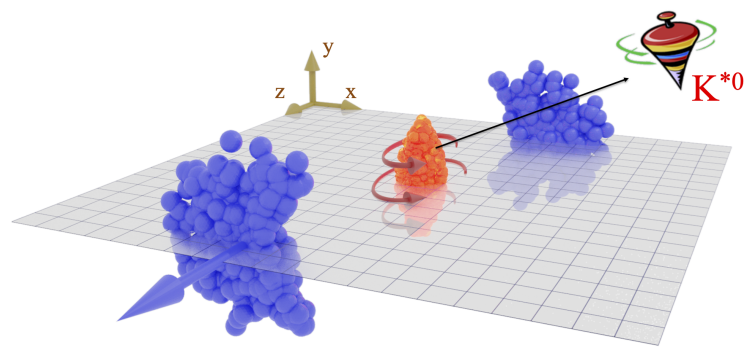

Figure 2: Illustration of a rotating quark-gluon plasma emitting a $\mathrm{K}_{0}^{*}$ meson with its spin aligned with the rotation of the emitting medium.

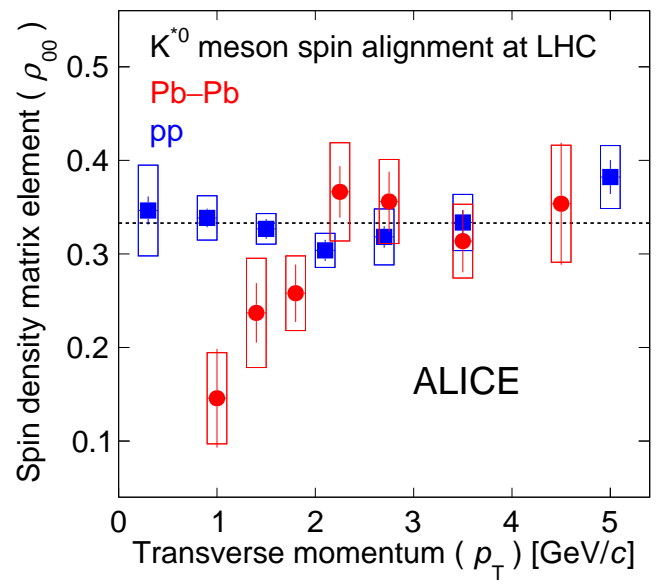

Figure 3: $\mathrm{K}_{0}^{*}$ spin density matrix element $\rho_{00}$ as a function of transverse momentum measured in $\mathrm{Pb}-\mathrm{Pb}$ collisions at $\sqrt{s_{\mathrm{NN}}}=2.76 \mathrm{TeV}$ and in proton-proton collisions at $\sqrt{s}=13 \mathrm{TeV}$. Adapted from [9].

as a whole rotates, as indicated in Fig. 2. As a consequence, the spin of vector mesons such as $\mathrm{K}^{* 0}$ is expected to be aligned with that motion, which is what is observed in measurements of the spin density matrix element $\rho_{00}$ in Pb-Pb collisions in Fig. $3[9,10]$. It is worth noting that the magnitude of the effect is significantly more pronounced than the $\Lambda$ polarization measurements by ALICE and STAR [11, 12], which poses an as-of-yet unresolved challenge to theorists.

The strongly interacting medium also blocks the passage of very-high momentum particles, leading to jet quenching. This effect can be quantified via the nuclear modification factor $R_{\mathrm{AA}}$, which is the ratio between the transverse momentum distributions observed in $\mathrm{Pb}-\mathrm{Pb}$ and an appropriately scaled spectrum measured in pp collisions at the same centre-of-mass energy. The $R_{\mathrm{AA}}$ has now been notably measured also for prompt $\mathrm{D}^{0}$ as well as non-prompt $\mathrm{D}^{0}$ mesons, with the latter coming from the decay of beauty hadrons [13]. While in both cases a strong suppression is observed in the $R_{\mathrm{AA}}$, as seen in Fig. 4 , the non-prompt $\mathrm{D}^{0}$ nuclear modification factor is slightly higher than the prompt $\mathrm{D}^{0}$ value between a transverse momentum of 5 and $10 \mathrm{GeV} / c$ [8], suggesting that beauty quarks undergo less quenching in the QGP than charm quarks. A potential explanation for this is the dead cone effect, according to which collinear gluon emissions are suppressed in $\theta<m_{q} / E_{q}$, where $\theta$ is the emission angle and $m_{q}$ and $E_{q}$ are the mass and energy of the emitting quark, respectively.

A more direct consequence of the dead cone effect has, in fact, also been observed in pp collisions at $\sqrt{s}=13 \mathrm{TeV}$, i.e. for the case of vacuum radiation. More specifically, the observable consequence of this effect is that charged-particle yield at small angles with respect to the jet axis are expected to be suppressed if the jet originated from a heavy-flavour quark. This effect can be seen very clearly in Fig. 5, in which the ratio of jet-associated particle yields is plotted as a function of the logarithm of the inverse of the opening angle for inclusive and for $\mathrm{D}^{0}$-tagged jets. It is visible that, at small opening angle $\theta$ (large values of $\ln (1 / \theta), \mathrm{D}^{0}$-tagged jets only exhibit half of the charged-particle yields than an inclusive jet sample [14]. 


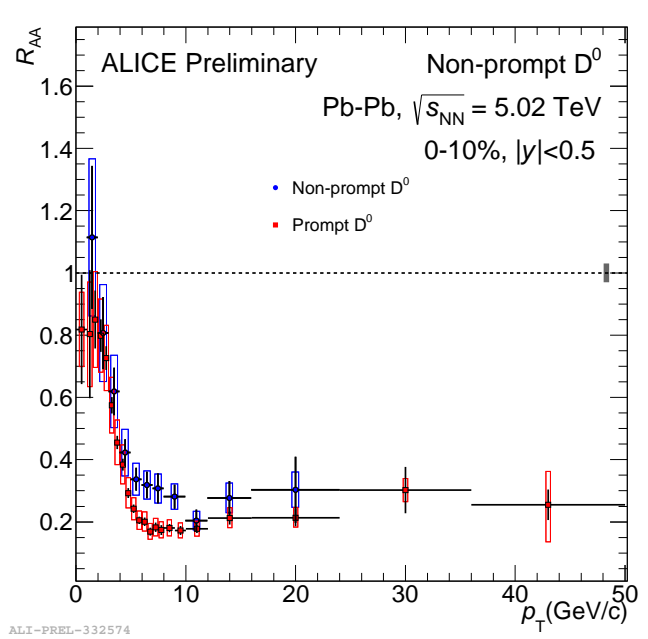

Figure 4: Nuclear modification factor $R_{\mathrm{AA}}$ as a function of transverse momentum for prompt and non-prompt $\mathrm{D}^{0}$ mesons in $\mathrm{Pb}-\mathrm{Pb}$ collisions at $\sqrt{s_{\mathrm{NN}}}=5.02 \mathrm{TeV}$.

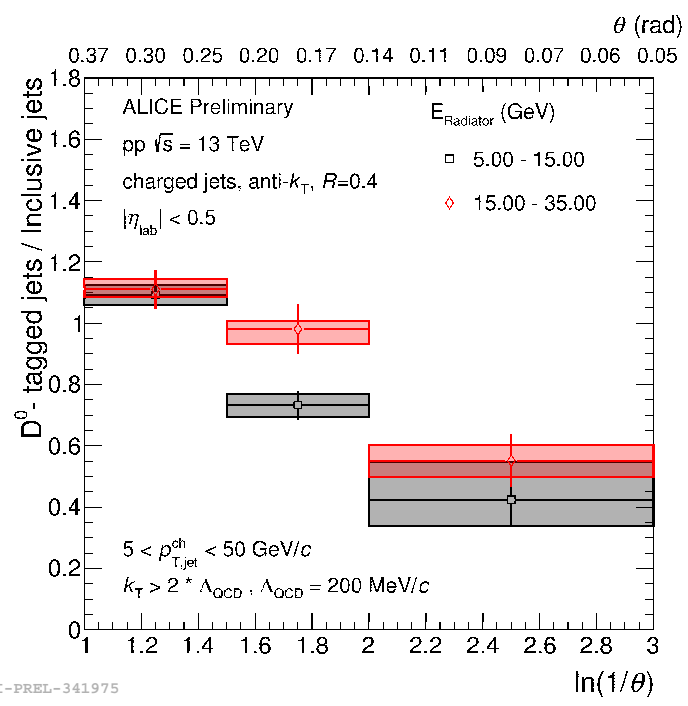

Figure 5: Ratio of charged-particle yields in $\mathrm{D}^{0}$-tagged and inclusive jets as a function of $\ln (1 / \theta)$, where $\theta$ is the angle with respect to the jet axis, as measured in $\mathrm{pp}$ collisions at $\sqrt{s}=13 \mathrm{TeV}$.

A detailed study of jet substructure has also been carried out in $\mathrm{Pb}-\mathrm{Pb}$ collisions at $\sqrt{s_{\mathrm{NN}}}=5.02$ $\mathrm{TeV}[15]$ and has revealed that in nucleus-nucleus collisions jets are more collimated than in pp at the same energy, as seen in Fig. 6. This measurement indicates that the medium has a significant resolving power for splittings with a particular dependence on the coherence scale.

\section{Emergence of QGP phenomena from QCD}

Substantial advances in our understanding of high density QGP were obtained in measurements performed in high-multiplicity pp and p-Pb collisions at the LHC. The observation of the double ridge [16-18] and strangeness enhancement [19] in such conditions were hallmark discoveries and their implications for heavy-ion physics is still being assessed by the community at large. To deepen our understanding of strangeness enhancement in proton-proton collisions, a double-differential study in which the $\Xi / \pi$ ratio is studied in low- and high-spherocity, high multiplicity pp collisions has been performed [20]. Figure 7 shows that more isotropic events, which present a larger spherocity, are also observed to have a larger $\Xi / \pi$ ratio, indicating that these are the ones that drive strangeness production higher and further constraining theoretical models.

Studies similar to the ones carried out in the light-flavour sector are also underway for heavyflavour hadrons such as the $\Lambda_{c}$ and the $\mathrm{D}^{0}$ meson [21]. These have been studied as a function of charged-particle multiplicity density in pp collisions at $\sqrt{s}=13 \mathrm{TeV}$, with the $p_{\mathrm{T}}$-differential $\Lambda_{c} / \mathrm{D}^{0}$ ratio exhibiting an increase at intermediate $p_{\mathrm{T}}$ that is reminiscent of that observed for the $\Lambda / \mathrm{K}_{\mathrm{S}}^{0}$, as can be seen in Fig. 8. When the $\Lambda_{c} / \mathrm{D}^{0}$ in a specific momentum interval is plotted as a function of the charged-particle multiplicity density in pp, $\mathrm{p}-\mathrm{Pb}$ and $\mathrm{Pb}-\mathrm{Pb}$, as seen in Fig. 9, the progression seems to be smooth as a function of multiplicity, again similarly to the $\Lambda / \mathrm{K}_{\mathrm{S}}^{0}$ [22]. When compared to PYTHIA8 simulations, the $\Lambda_{c} / \mathrm{D}^{0}$ can only be qualitatively described when 


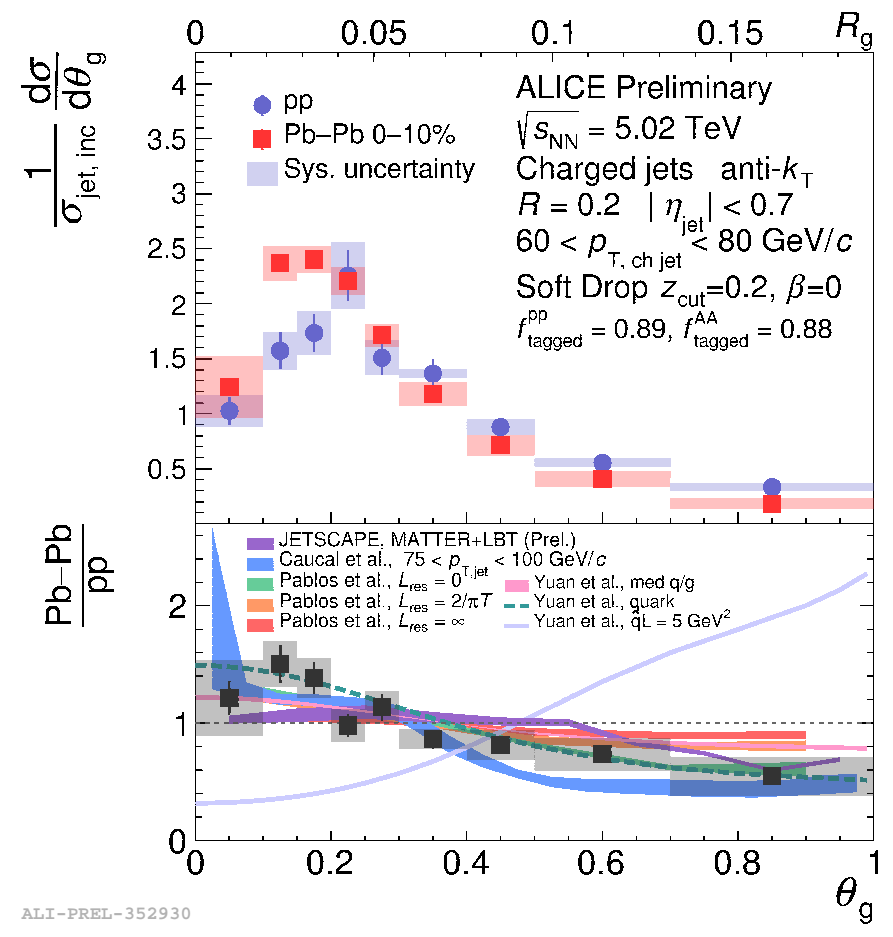

Figure 6: Distribution of first jet splitting angle $\theta_{g}$ measured in $\mathrm{Pb}-\mathrm{Pb}$ collisions at $\sqrt{s_{\mathrm{NN}}} \mathrm{TeV}$ and in pp collisions at the same energy using jet grooming techniques. Figure from [15].

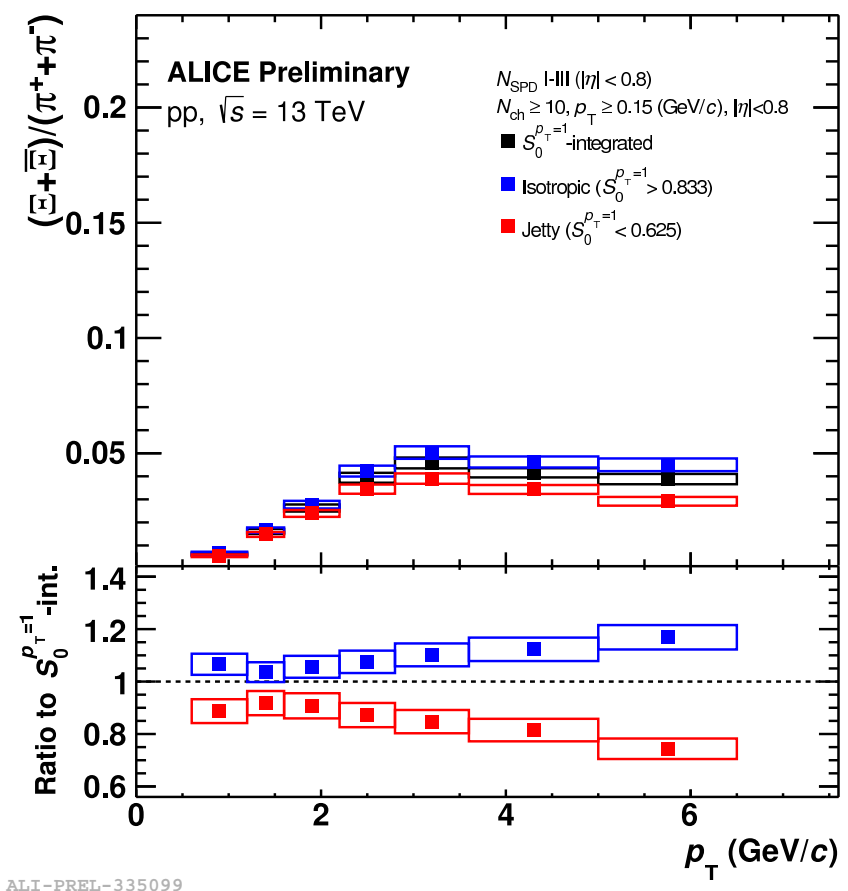

Figure 7: Transverse-momentum-differential $\Xi / \pi$ ratio in high- and low-spherocity pp collisions at $\sqrt{s}=13$ $\mathrm{TeV}$. 


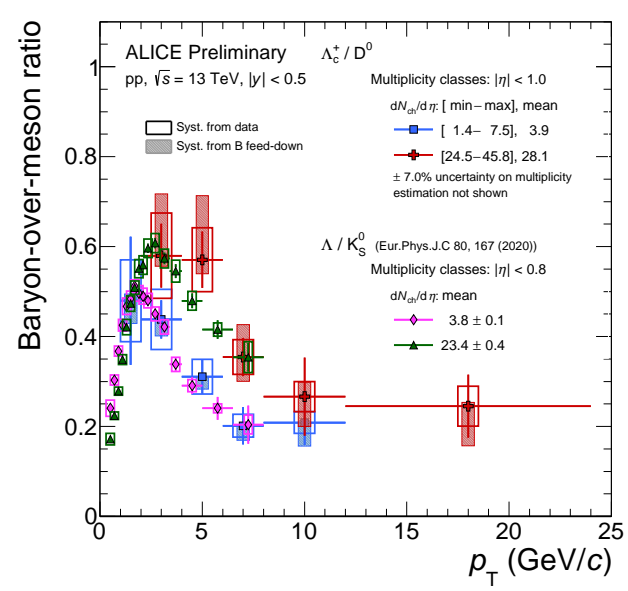

Figure 8: Transverse-momentum-differential $\Lambda_{c}^{+} / \mathrm{D}^{0}$ ratio as a function of charged-particle multiplicity density in pp collisions at $\sqrt{s}=13$ $\mathrm{TeV}$ shown together with the $\Lambda / \mathrm{K}_{\mathrm{S}}^{0}$ ratio for comparison.

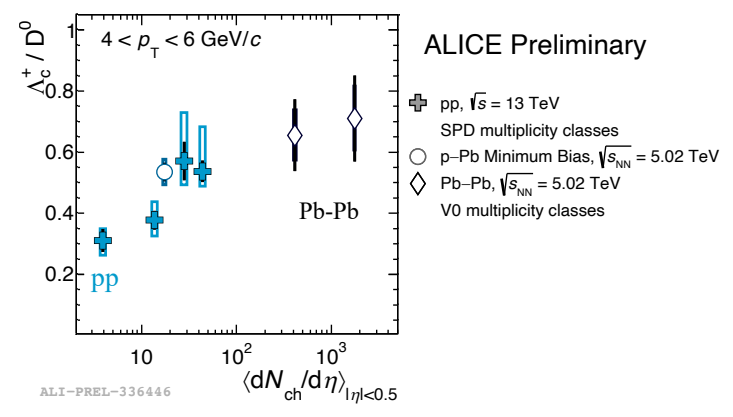

Figure 9: Multiplicity dependence of the $\Lambda_{c}^{+} / \mathrm{D}^{0}$ ratio in the momentum interval $4.0<p_{\mathrm{T}}(\mathrm{GeV} / c)<6.0$ in pp collisions at $\sqrt{s}=13 \mathrm{TeV}$ and in $\mathrm{p}-\mathrm{Pb}$ and $\mathrm{Pb}-\mathrm{Pb}$ collisions at $\sqrt{s_{\mathrm{NN}}}=5.02 \mathrm{TeV}$.

using color reconnection in 'mode 2', in which colour junctions are allowed to form [23], as can be seen in Fig. 10. Further measurements in Run 3 will allow for a significant reduction in both statistical and systematic uncertainties in this measurement [24].

Similar observations also hold for the $p_{\mathrm{T}}$-differential $\Sigma_{c}^{0,+,++} / \mathrm{D}^{0}$ and $\Xi_{c}^{0,+} / \mathrm{D}^{0}[26]$, though for the latter even PYTHIA with junctions fails to reproduce the data by more than a factor of three. The $\Xi_{c}^{0,+} / D^{0}$, shown in Fig. 11, is also not correctly reproduced by a coalescence model [25]. These findings indicate that a lot is still to be understood regarding charmed baryon production in high-energy collisions, with more insights expected to come from the LHC Run 3 and beyond.

\section{Beyond heavy ions: The LHC as a general-purpose QCD laboratory}

The excellent particle identification capabilities of the ALICE experiment, coupled with the copious production of hadrons at the LHC, leads to unique opportunities for advancing our understanding of QCD in general. One example of a study that leveraged these conditions is the first measurement of antideuteron and antihelium absorption [27], which led to cross section measurements at low momenta that are highly relevant to astrophysics. The preliminary result for the ${ }^{3} \overline{\mathrm{He}}$ is shown in Fig. 12 and the corresponding result for antideuterons is published in [28]. Further advances in antinuclei measurements are expected in Run 3 and beyond.

Another very exciting opportunity that has presented itself at the LHC is to study the protonhyperon interaction. This can be performed by measuring proton-hyperon pair momentum correlations, which, combined with a determination of the particle-emitting source size, allows for a precise comparison of the interaction characteristics to predictions from lattice $\mathrm{QCD}$, as can be seen in Fig. 13. This measurement, published in Nature [29], further adds to our understanding of proton-hyperon interactions, which are relevant for constraining the equation of state of neutron 


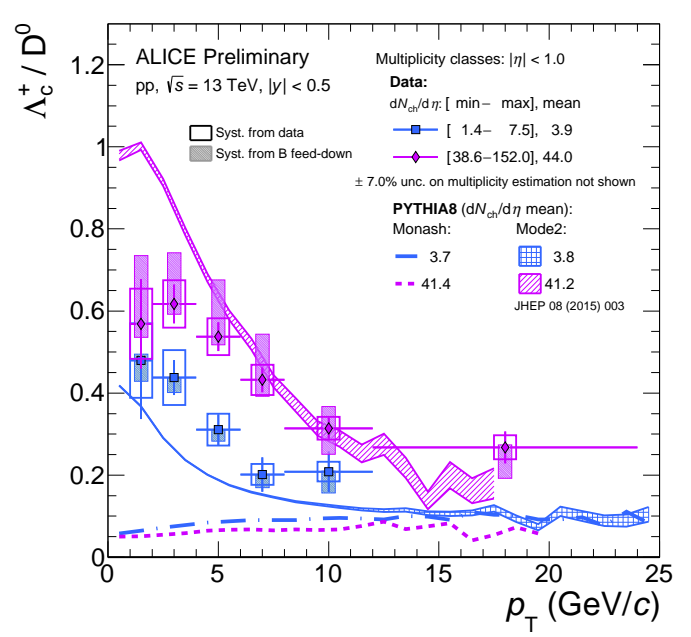

Figure 10: Transverse-momentum-differential $\Lambda_{c}^{+} / \mathrm{D}^{0}$ ratio in two selected charged-particle multiplicity intervals in pp collisions at $\sqrt{s}=13 \mathrm{TeV}$ compared to predictions from PYTHIA8 using various settings of colour reconnection.

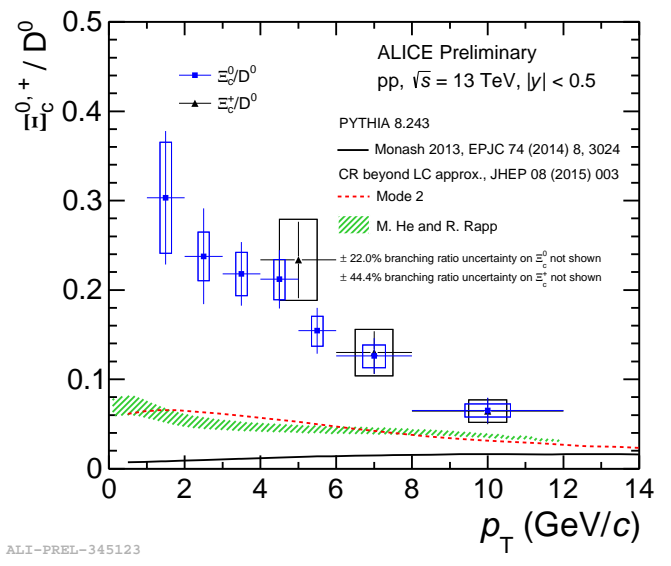

Figure 11: Transverse-momentum-differential $\Xi_{c}^{0,+} / \mathrm{D}^{0}$ ratio in two selected charged-particle multiplicity intervals in pp collisions at $\sqrt{s}=13 \mathrm{TeV}$ compared to predictions from PYTHIA8 using various settings of colour reconnection as well as with predictions from the coalescence model from M. He and R. Rapp [25].

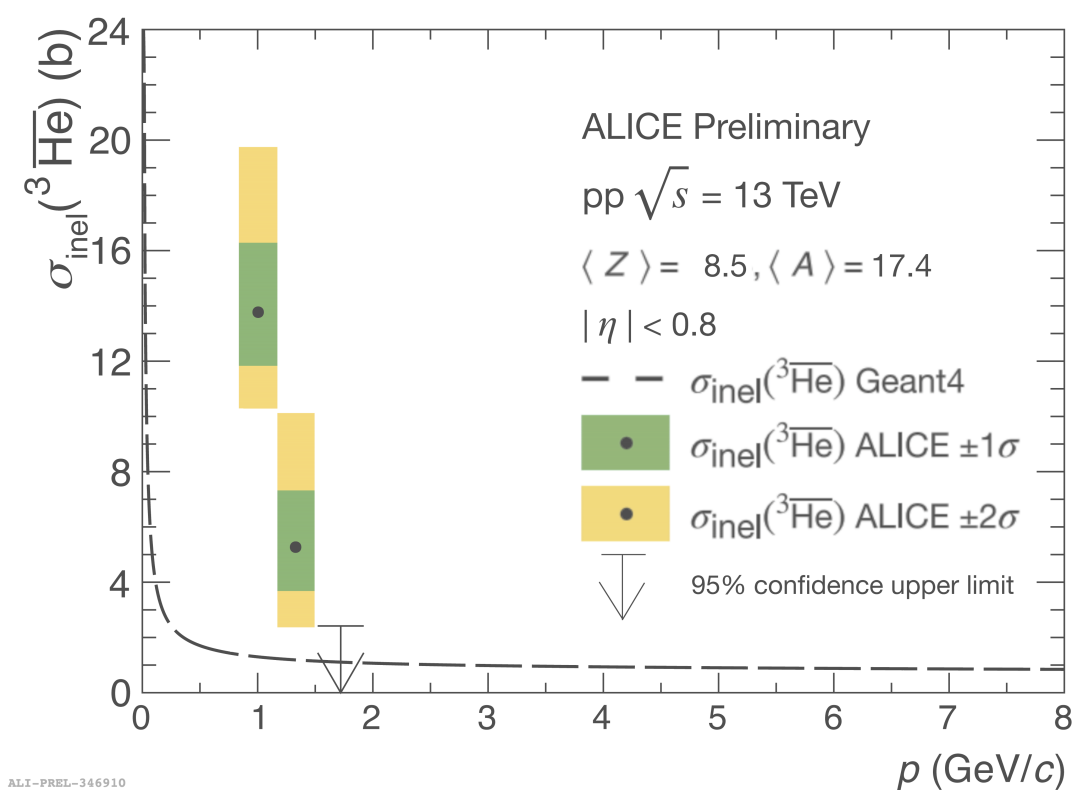

Figure 12: Inelastic cross section $\sigma_{\text {inel }}\left({ }^{3} \overline{\mathrm{He}}\right)$ as a function of total momentum measured via secondary interactions with the material in pp collisions at $\sqrt{s}=13 \mathrm{TeV}$. 


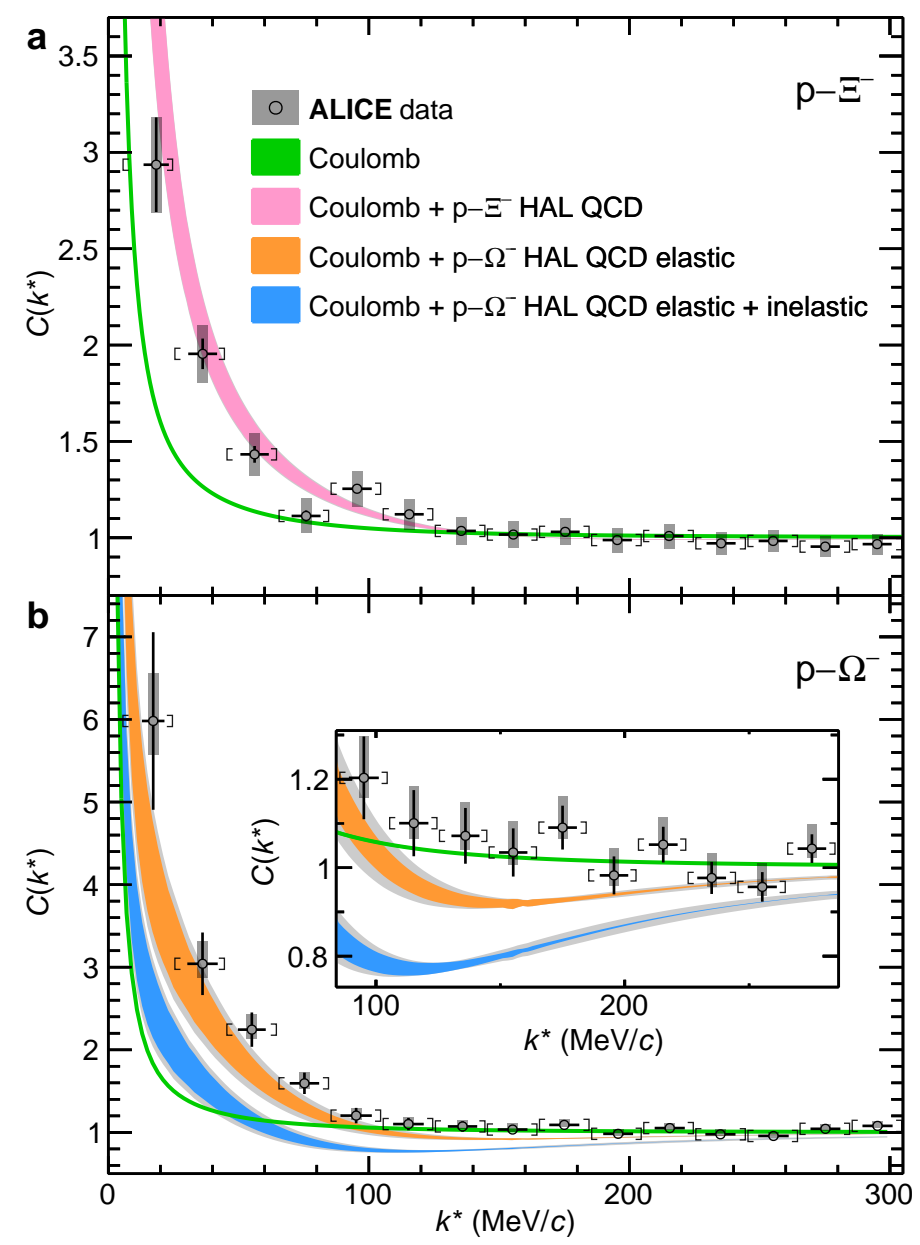

Figure 13: Experimental $\mathrm{p}-\Xi^{-}$and $\mathrm{p}-\Omega^{-}$correlation functions measured in high-multiplicity pp collisions at $\sqrt{s}=13 \mathrm{TeV}$ compared to predictions from lattice QCD using various combinations of interaction terms. Figure from [29].

stars, and constitutes the first measurement of an attractive strong interaction between protons and $\Omega$ baryons.

\section{The bright future ahead}

The ALICE collaboration has a comprehensive upgrade programme foreseen in the next years. During Long Shutdown 2 (LS2), the TPC readout is being replaced in its entirety by a GEM-based system that is able to cope with a factor 50 higher data rate. Furthermore, the inner tracking system (ITS), previously comprised of pixel, strip and drift detectors, is being replaced by a fully pixel-based system, effectively multiplying the number of channels by a factor of a thousand. The much improved ITS, coupled with a pixel-based muon forward tracker (MFT), will improve impact parameter resolution by a factor of 3 in Run 3. This detector setup will lead to significant advances in our understanding of heavy-flavour meson and baryon production, as well as enable studies of 
(anti)(hyper)nuclei up to a mass number of 4 . In addition, hadronic interactions up to $\Omega-\Omega$ pairs will be within reach [30], and a first measurement of the low-mass dielectron spectrum will allow for an estimate of the QGP temperature before hadronization.

Further into the future, the Long Shutdown 3 will also bring about the installation of an even more advanced inner tracking system, the ITS3 [31], comprised solely of thin bent sheets of silicon in the active detection area. This groundbreaking detector will enable even higher precision studies of low- $p_{\mathrm{T}}$ charm production, including the possibility of searching for charmed nuclei. In addition, a forward calorimeter, FoCal, is planned, and will allow for high-precision measurements of the gluon nPDF down to $x \approx 10^{-5}$ and below [32].

Even beyond, a project for a next-generation heavy-ion experiment at the LHC, named ALICE 3 , is being actively pursued [33]. It is to be a fast, ultra-thin detector solely based on silicon sensors, allowing for yet another fifty-fold increase in data rates. In so doing, ALICE 3 will be able to exploit higher LHC luminosities using nuclei lighter than lead, while still retaining particle identification capabilities via state-of-the-art silicon-based time of flight determination with $\sim 20 \mathrm{ps}$ time resolution. This brand new detector will enable ultimate performance for multi-heavy-flavour hadron detection, thermal radiation via virtual photons from the QGP and soft hadrons (below $p_{\mathrm{T}}$ of $50 \mathrm{MeV} / \mathrm{c}^{2}$ ). This initiative has been supported in the latest European Strategy for Particle Physics Update, ensuring that the future of heavy-ion physics will remain strong even beyond the next decade.

\section{Acknowledgements}

The author would like to acknowledge support from FAPESP via project number 17/05685-2 and CNPq via grant number 312661/2019-3.

\section{References}

[1] F. Antinori and U. Wiedemann, ALICE: Physics Performance Report, Volume I, Journal of Physics G: Nuclear and Particle Physics 30 (2004) 1515.

[2] ALICE collaboration, Measurement of the (anti-) ${ }^{3}$ He elliptic flow in Pb-Pb collisions at $\sqrt{s_{\mathrm{NN}}}=5.02 \mathrm{TeV}$, Phys. Lett. B 805 (2020) 135414 [1910.09718].

[3] ALICE collaboration, Elliptic and triangular flow of (anti)deuterons in Pb-Pb collisions at $\sqrt{s_{\mathrm{NN}}}=5.02 \mathrm{TeV}$, Phys. Rev. C 102 (2020) 055203 [2005.14639].

[4] V. Vislavicius, Constraining the transport properties of QGP with latest flow measurements, These proceedings .

[5] R. Derradi de Souza, T. Koide and T. Kodama, Hydrodynamic approaches in relativistic heavy ion reactions, Progress in Particle and Nuclear Physics 86 (2016) 35-85.

[6] ALICE collaboration, J/ $\psi$ elliptic and triangular flow in Pb-Pb collisions at $\sqrt{s_{\mathrm{NN}}}=5.02 \mathrm{TeV}, \mathrm{JHEP} 10$ (2020) 141 [2005. 14518].

[7] ALICE collaboration, Elliptic flow of electrons from beauty-hadron decays in $\mathrm{Pb}$-Pb collisions at $\sqrt{s_{\mathrm{NN}}}=5.02$ $\mathrm{TeV}, 2005.11130$.

[8] X. Peng, Beauty production and anisotropy with ALICE at the LHC, These proceedings .

[9] ALICE collaboration, Evidence of Spin-Orbital Angular Momentum Interactions in Relativistic Heavy-Ion Collisions, Phys. Rev. Lett. 125 (2020) 012301 [1910. 14408].

[10] B. Mohanty, Spin alignment measurements of vector mesons, These proceedings .

[11] ALICE collaboration, Global polarization of $\Lambda \bar{\Lambda}$ hyperons in Pb-Pb collisions at $\sqrt{s_{N N}}=2.76$ and $5.02 \mathrm{TeV}$, Phys. Rev. C 101 (2020) 044611 [1909.01281].

[12] STAR collaboration, Global $\Lambda$ hyperon polarization in nuclear collisions: evidence for the most vortical fluid, Nature 548 (2017) 62 [1701.06657]. 
[13] J. Mulligan, Overview of the latest jet physics results from ALICE, These proceedings .

[14] L. Cunqueiro, First direct measurement of the dead-cone effect at colliders, These proceedings .

[15] ALICE collaboration, Physics Preliminary Summary: Measurements of the groomed jet radius and groomed momentum fraction in pp and Pb-Pb collisions at $\sqrt{s_{\mathrm{NN}}}=5.02 \mathrm{TeV}$, ALICE-PUBLIC-2020-006 (2020) .

[16] ATLAS collaboration, Observation of Associated Near-Side and Away-Side Long-Range Correlations in $\sqrt{s_{\mathrm{NN}}}=$ $5.02 \mathrm{TeV}$ Proton-Lead Collisions with the ATLAS Detector, Physical Review Letters 110 (2013) .

[17] ALICE collaboration, Long-range angular correlations on the near and away side in $p-P b$ collisions at $\sqrt{s_{\mathrm{NN}}}=$ 5.02 TeV, Physics Letters B 719 (2013) 29 .

[18] CMS collaboration, Observation of long-range, near-side angular correlations in proton-proton collisions at the LHC, JHEP 2010 (2010) .

[19] ALICE collaboration, Enhanced production of multi-strange hadrons in high-multiplicity proton-proton collisions, Nature Phys. 13 (2017) 535 [1606.07424].

[20] S. Tripathy, Topological studies of light-flavour hadron production in high-multiplicity pp collisions, These proceedings .

[21] L. Vermunt, Open heavy-flavour production from small to large collision systems with ALICE at the LHC, These proceedings .

[22] ALICE collaboration, Multiplicity dependence of light-flavor hadron production in pp collisions at $\sqrt{s}=7 \mathrm{TeV}$, Phys. Rev. C 99 (2019) 024906 [1807.11321].

[23] J.R. Christiansen and P.Z. Skands, String Formation Beyond Leading Colour, JHEP 08 (2015) 003 [1505 . 01681$].$

[24] ALICE collaboration, Future high-energy pp programme with ALICE, Tech. Rep. ALICE-PUBLIC-2020-005.

[25] M. He and R. Rapp, Charm-Baryon Production in Proton-Proton Collisions, Phys. Lett. B 795 (2019) 117 [1902.08889].

[26] J. Zhu, Hadronization studies with ALICE, These proceedings .

[27] S.A. Konigstorfer, Measurement of the anti-nuclei nuclear inelastic cross sections with ALICE and implications for indirect Dark Matter searches, These proceedings .

[28] ALICE collaboration, Measurement of the low-energy antideuteron inelastic cross section, Phys. Rev. Lett. 125 (2020) 162001 [2005.11122].

[29] ALICE collaboration, Unveiling the strong interaction among hadrons at the LHC, Nature 588 (2020) 232-238.

[30] Z. Citron et al., Report from Working Group 5: Future physics opportunities for high-density QCD at the LHC with heavy-ion and proton beams, CERN Yellow Rep. Monogr. 7 (2019) 1159 [1812.06772].

[31] L. Musa, Letter of Intent for an ALICE ITS Upgrade in LS3, Tech. Rep. CERN-LHCC-2019-018. LHCC-I-034, CERN, Geneva (Dec, 2019).

[32] ALICE collaboration, Letter of Intent: A Forward Calorimeter (FoCal) in the ALICE experiment, Tech. Rep. CERN-LHCC-2020-009. LHCC-I-036, CERN, Geneva (Jun, 2020).

[33] D. Adamová et al., A next-generation LHC heavy-ion experiment, 1902.01211. 